\title{
SPACE QUALIFIED MAGNETIC DISK
}

\author{
Arthur J. Treff \\ John F. Forella \\ Raymond Engineering, Inc. \\ Memory Systems Division \\ Middletown, CT 06457
}

\section{KEYWORDS}

Magnetic Disk, Space Environment

\begin{abstract}
Highly reliable data storage for satellites and spacecraft is a challenging technology. The space environment is a unique combination of many environmental factors which impact the reliability and even the very survival of electronic systems. The need for space qualified memory is becoming even more important with the advent of on-board data processing which requires rapid access to large data bases.

This paper describes the unique environmental and design considerations that must be taken into account for a magnetic disk that is designed to operate for years in the space environment.
\end{abstract}

\section{INTRODUCTION}

Magnetic hard disks have been the media of choice in commercial mass storage systems for many years. Driven by the surging demands of the work station and personal computer marketplace, magnetic disks have reached a high level of sophistication and have maintained a remarkable capacity growth. Unfortunately, commercial hard disks have also maintained the reputation as rather fragile devices. Although early designs were plagued by head crash problems, today the typical Winchester disk is quite reliable in the office environment. Small form factor drives are also enjoying success in the portable lap-top and notebook environment.

A magnetic disk which is designed for use in space takes advantage of the sophistication of the state of the art in commercial drives but must build upon this to 
develop a device which is unique and takes into consideration the particular environmental and performance requirements.

The application of magnetic disk in the space environment is a highly specialized endeavor which involves many disciplines. The device must be robust enough to survive the mechanical vibration and shock of the launch vehicle. It must operate for long periods of time in a vacuum with high and low temperature extremes and it must survive the natural radiation of space without upset, latch-up or performance degradation.

The overall design of the magnetic disk must also take into account some unique design considerations. All materials must be chosen with material outgassing as a consideration. The design must also provide an operational life of several years of unattended operation. The reliability of the parts used in the design must meet space requirements and the qualified design configuration must be controlled to ensure continued reliability through the inevitable component and manufacturing process changes.

\section{MEMORY TECHNOLOGY CONSIDERATIONS}

This paper is based on magnetic disk technology. It will not address the memory technology trade-offs which go into the selection of magnetic disk for the space application. However, recognizing that magnetic disks offer major advantages in terms of non-volatility, capacity, speed, volumetric efficiency and cost, it is assumed in this paper that magnetic disks are the media of choice. A trade-off analysis would compare magnetic disk to the cost, life and radiation limitations of Flash EPROM, the cost and volatility of rad-hard SRAM and the mechanical complexity, reliability and limited capacity of optical disk.

\section{SPACECRAFT ENVIRONMENTAL CONSIDERATIONS}

The unique combination of environmental factors encountered by spacecraft and satellites mandate a unique design. The environmental conditions start with the shock, vibration and acceleration of the launch vehicle and continue with the temperature, vacuum, angular acceleration and radiation in space.

Launch Vehicle Environment - The magnetic disk must survive the launch into space without damage. The shock, vibration and acceleration experienced during the launch and ascent phases is dependent on the launch vehicle used. The typical vehicles are Titan, Delta, Atlas and the Space Shuttle. The environmental performance required is summarized below. 
Launch Shock

Launch Vibration

Pyro Shock (Stage Separation)

Launch Acceleration
15 G@8 Milliseconds Sawtooth

$15.3 \mathrm{Grms}$

$3000 \mathrm{G} @ .25$ Milliseconds

$12 \mathrm{G}$

The key to immunity to the mechanical inputs of shock, vibration and acceleration is the balancing of all moving components. The head actuator must be triaxially balanced which then nulls all acceleration inputs and allows the drive to ride through high levels of shock and vibration with no impact. In addition, the mass of these components must be kept to an absolute minimum which further minimizes the acceleration effects and has the added benefit of minimizing power consumption and access time.

Angular Acceleration - The magnetic disk must be able to operate on a spinning spacecraft where the resultant angular acceleration is several $\mathrm{G}^{\prime} \mathrm{s}$. This requirement is in further support of a totally balanced design. Additionally, head actuator motors must be of high enough torque and their controlling servos of wide enough bandwidth to maintain data access in this high $\mathrm{G}$ environment. The magnetic disk is then totally immune to the effects of acceleration (angular or linear) and conversely is perfectly happy operating in an absence of any acceleration at all (zero G).

By the same token, the magnetic disk must not import angular acceleration on the spacecraft by its own momentum during disk spin-up. This effect is counteracted by mounting two disk drives back to back and counter rotating them simultaneously. The back to back disk drives have the added benefit of redundancy.

Thermal/Vacuum - The on-orbit thermal environment combined with the effects of a vacuum require a thermal management system based on a conductive thermal path to a cold plate to remove all internally generated heat. A major advantage of the uniquely designed system is the separation of the disk drive mechanism from the controlling electronics within the box. The disk drives are mounted on shock isolators for good vibration and shock performance, but these isolators provide poor thermal conduction. The heat generating control circuitry is therefore, hard mounted separately with excellent thermal conduction characteristics. Extensive use of thermal ladders in the control circuitry and generous conduction surfaces provide a reliable thermal design.

The thermal/vacuum environment is summarized below.

$\begin{array}{ll}\text { On-Orbit Thermal } & -20^{\circ} \mathrm{C} \text { to }+60^{\circ} \mathrm{C} \\ \text { On-Orbit Vacuum } & 1 \times 10^{-7} \text { Torr }\end{array}$


Natural Radiation - The magnetic disk must be able to operate in and survive the natural radiation environment of high inclination orbits. The two radiation effects of concern are Single Event Effects and Total Dose Effects. The magnetic media itself is immune to the radiation. Only the semiconductor control circuitry is effected.

Galactic cosmic radiation results in the phenomenon of Single Event Effects (SEE) in semiconductors. Individual electronic devices are upset (SEU) or latch-up (SEL) as a result of this radiation. The specific technology of the semiconductor device and the density of the solid state circuitry are the keys to its susceptibility to this type of radiation.

The hardening procedure to natural radiation for the magnetic disk requires that an SEE pedigree be determined for each and every electronic part in the system. An extensive radiation data search is made and devices with no data are SEE tested at an appropriate lab to determine susceptibility. Devices which upset too frequently or latch-up are not used. Single Event Upset recovery is also incorporated into the design to provide an orderly recovery should an upset occur.

The total dose effects also require the establishment of a pedigree for each part. Those parts which cannot operate for long periods of time in space without degradation or burn-out are not used.

The basic radiation environment is summarized below.

Total Dose

SEE
$1 \times 10^{5}$ Rads ( $\left.\mathrm{Si}\right)$

$1 \times 10^{10}$ error/bit-day SEU

\section{SYSTEM DESCRIPTION}

The Raymond Engineering Space Qualified Magnetic Disk is an adaption of a previously developed, fully militarized design currently fielded on several airborne military applications (see Figure 1). The 40 megabyte version has attained over 30,000 failure free operating hours on the F-117 Stealth Fighter aircraft. The 300 megabyte version is currently being integrated into the Multifunction Electronic Display System (MEDS) for the Space Shuttle cockpit.

These products are uniquely designed systems specifically developed for severe environment applications. They have several unique features that make them well suited for those environments. 
*Oversized spindle motor and conservative low stress spindle bearing.

*Totally balanced head actuator with automatic head retraction and parking off the media.

*Hermetically sealed disk cartridge filled with dry gas.

*Extensive Built-In-Test and self diagnostic capability.

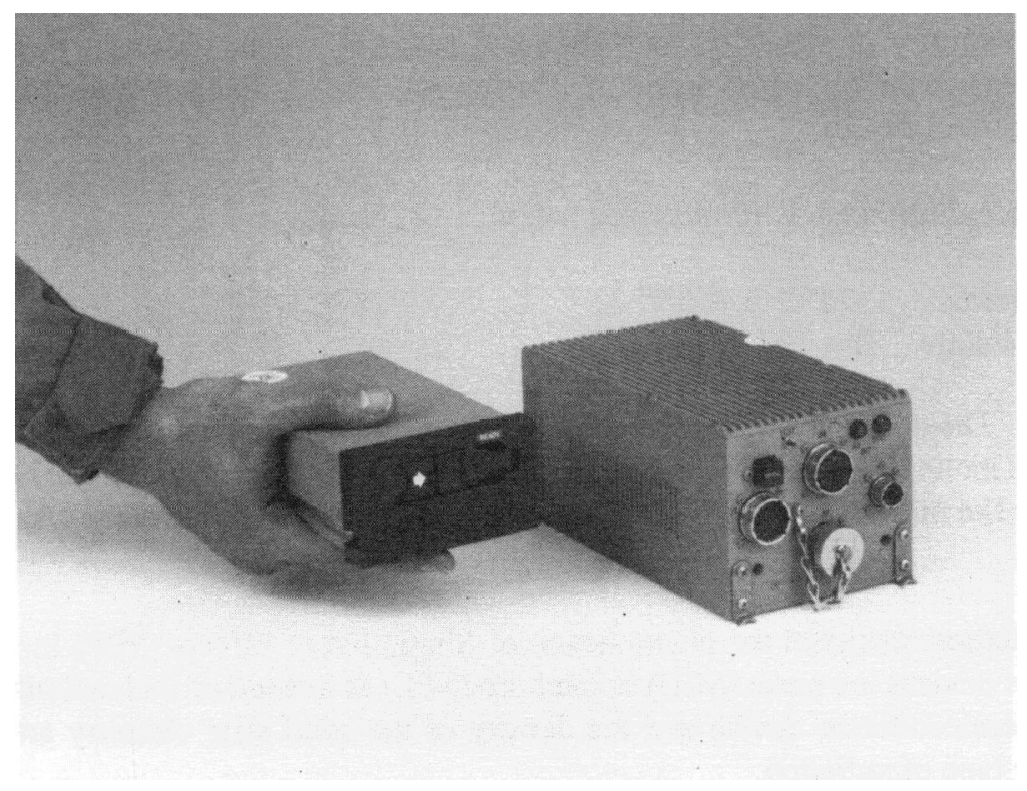

Figure 1. 300 Mbyte Magnetic Disk for Severe Environment Applications

The Space Qualified Magnetic Disk is a 2 Gigabyte (16 Gigabit) growth version of the previous design and is developed specifically for space applications (See figure 2). It is a small, lightweight device incorporating two hermetically sealed, 1 Gigabyte disk cartridges mounted in a counter rotating configuration. The disk drive control and interface electronics are mounted separately in the same enclosure and incorporate conductive thermal management.

The system interface is via SCSI (Small Computer System Interface) whose bus structure allows easy growth with the expansion of additional units.

The space qualified system design incorporates all of the aforementioned features necessary for the spacecraft environment. Several other design considerations, unique to the space environment, are also incorporated.

Material Outgassing - All materials and finishes are evaluated for outgassing properties and suitability for use on spacecraft. A detail material survey is made and 
compared with the appropriate NASA requirements. Inappropriate materials are not used.

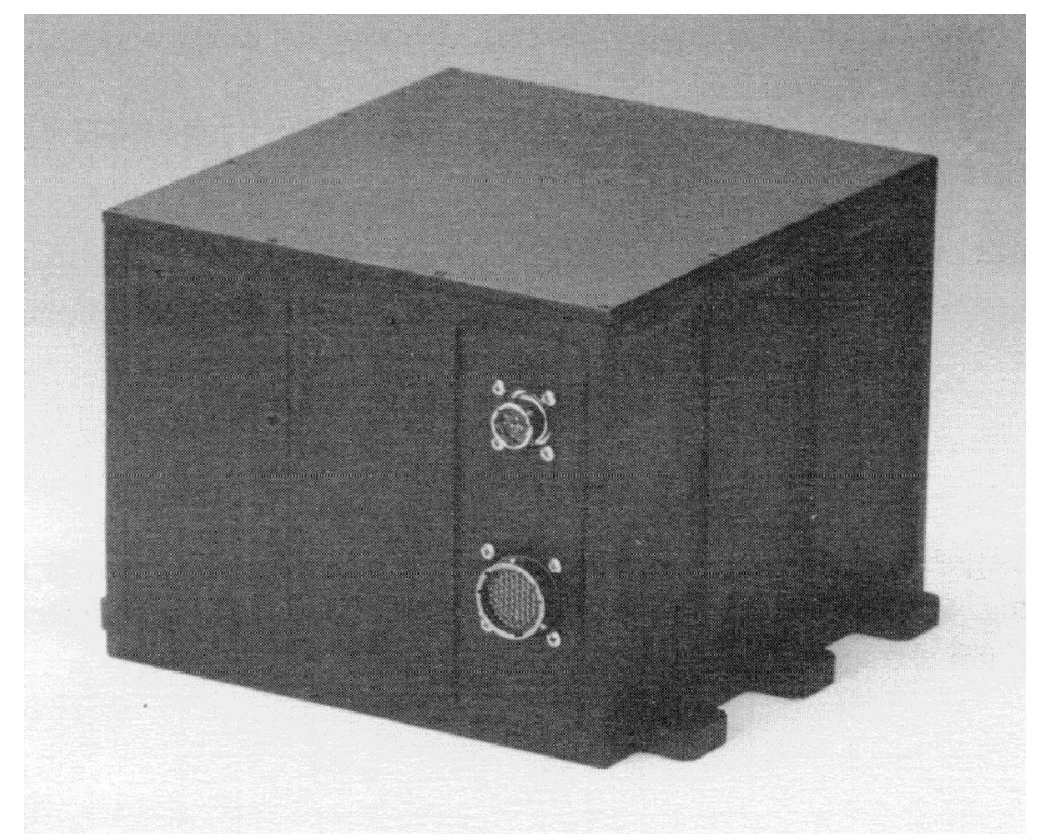

Figure 2. Raymond Engineering's Space Qualified Magnetic Disk

Operational Life - Unattended operation for long periods of time is a key requirement for a memory device in space. Magnetic disk media life is inherently unlimited, however, the mechanical drive mechanism does have life limits. The number of moving parts in the mechanism is kept to a minimum but the component with the shortest life, by far, is the spindle bearing.

The key to spindle life performance is a proprietary bearing design and lubrication with a unique bearing preload technique. This unique bearing has demonstrated a continuous operating life of several years.

The operational life of the magnetic disk is further increased with the implementation of an automatic duty cycle operation where the disk is spun down if it is not accessed for long periods of time.

Space Class parts - The Space Qualified Magnetic Disk incorporates Space Class Electronic Components to the largest extent possible.

Configuration Control - The Space Qualified Magnetic Disk configuration, resulting from the spacecraft environmental considerations and the unique design considerations, must be continuously monitored and maintained. 


\section{SUMMARY}

A magnetic disk designed specifically for application in a space environment is available today from Raymond Engineering. Space based systems can now utilize the low mass, high data transfer rate, fast access times, high capacity and low cost of magnetic disks. 